\title{
Juxtarenal Aortic Aneurysm Treated with Chimney Technique
}

\section{Beytullah Cakal, Sinem Deniz Cakal, Oguz Karaca*, Onur Omaygenc and Bilal Boztosun}

Medipol University Faculty of Medicine, Cardiology Department, Istanbul, Turkey

Keywords: Endovascular aneurysm repair; Abdominal aortic aneurysm

\section{Introduction}

Endovascular aneurysm repair (EVAR) compares favorably to open surgical repair of abdominal aortic aneurysm (AAA). For anatomically challenging aortic aneurysms such as inadequate sealing zone, chimney graft stent has been developed involving renal, visceral or internal iliac branches by placing parallel stents to the main stent graft to preserve blood flow to aortic branches [1,2]. Early and mid-term results of chimney technique have reported similar-in hospital mortality and less morbidity than surgical treatment but long term data also needs to be determined for concerns about patency and endoleak $[3,4]$.

\section{Description}

A 78-year-old man with a history of hypertension and abdominal pain was referred to our hospital for a palpable mass in the abdomen suggesting AAA. Computed tomography angiography (CTA) demonstrated a partially thrombosed AAA with a maximum diameter of $77 \mathrm{~mm}$ extending to the right common iliac artery measuring $55 \mathrm{~mm}$ and an angulated proximal aortic neck of $26 \mathrm{~mm}$ in diameter (Figure $1 \mathrm{~A}$ and $1 \mathrm{~B}$ ). Although the length of aortic aneurysm was $6 \mathrm{~mm}$ to the right renal artery, aneurysm involved left renal artery. Therefore, conventional EVAR would compromise left renal blood flow, we decided to perform EVAR using the chimney technique for AAA.

Vascular access in both femoral arteries was obtained. The left renal artery was cannulated using a $4 \mathrm{Fr}$ vertebral $120 \mathrm{~cm}$ catheter through $6 \mathrm{Fr} 90 \mathrm{~cm}$ (telescopic technique) destination sheath (Terumo) with a 0.035 " wire (Amplatz stiff wire Cook Medical Inc. Bloomington, IN, USA) via left brachial artery. An $8 \times 59 \mathrm{~mm}$ graft stent (Advanta Vascular V12, Atrium Europe B.V. Mijdrecht, Netherlands) was placed into the left renal artery along the wire. A $36 \times 20 \times 170 \mathrm{~mm}$ aortic extension stent graft (Endurant, Medtronic) was positioned just below the right renal artery through the left femoral access and deployed (Video 1). The ostium of the left renal artery was covered by the graft. A final kissing balloon was achieved (Figure 2A). Since the aneurysm extended to right common iliac artery a $16 \times 13 \times 199$ graft stent (Endurant 2, Medtronic) was positioned and deployed in overlap with the distal part of the graft stent and post-dilated using compliant Reliant balloon (Medtronic). Post-procedure angiography showed type $1 \mathrm{~A}$ endoleak and patent left renal artery. After second kissing balloon inflation a minimally residual endoleak was observed (arrowhead, Figure 2B, Video 2). Following the procedure, bilateral femoral sheaths were removed and hemostasis was achieved by deployment from the predelivered perclose Proglide (Abbott Vascular). The patient was discharged without complication on second day on admission. Post procedural CTA showed no endoleak at first month follow-up (Figure 3). The patient is now under regular follow-up without any symptoms.

Compared to fenestrated or branched stent, chimney stent has the advantage of avoiding the delay in device preparation and supply and relatively lower cost. In Turkey, for example, the price of a fenestrated device is currently about $€ 20,000$ compared to that $€ 5000$ of chimney graft stent. The endovascular surgery takes about 4-6 hours and the patient needs 5-7 weeks to recover from aneurysm surgery, after 5-7 days of hospital stay, whereas our patient was discharged from hospital on second day after the procedure.

Since short aortic neck, angulation, inappropriate sealing zone restrict the general applicability of EVAR, chimney method is cheaper, immediately available procedure in high risk patients whose renal, mesenteric or internal iliac arteries are jeopardized in the EVAR process

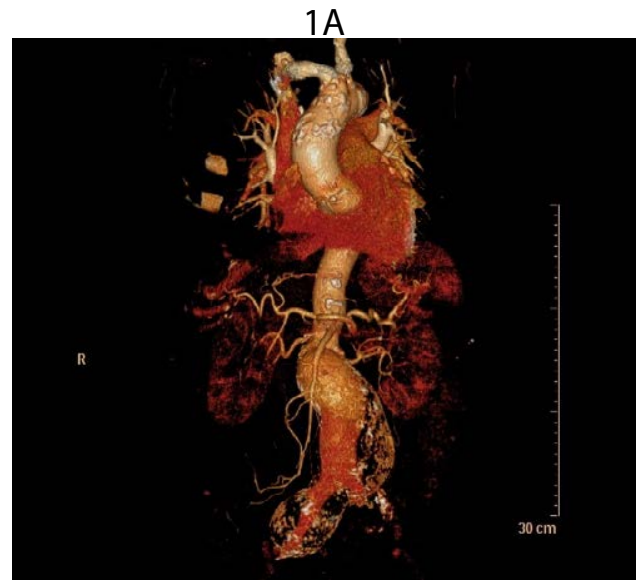

$1 \mathrm{~B}$

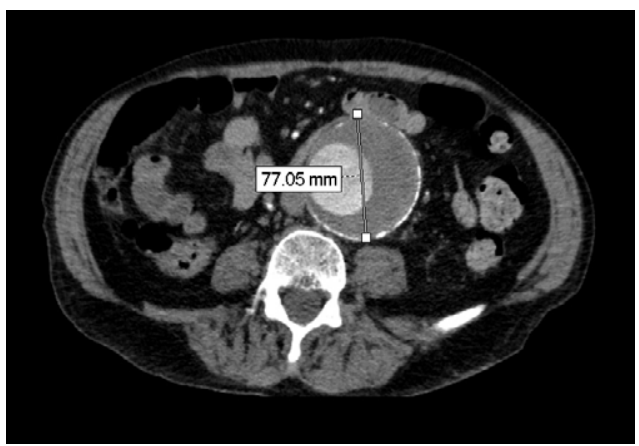

Figure 1 A,B: Baseline CT images of abdominal aortic aneurysm CT showed partially thrombosed abdominal aortic aneurysm with a maximum diameter of $78 \mathrm{~mm}$ with an angulated proximal aortic neck (ca. $80^{\circ}$ ) of $26 \mathrm{~mm}$ in diameter extending down the right common iliac artery.

*Corresponding author: Oguz Karaca, Medipol University Faculty of Medicine Cardiology Department, Istanbul, Turkey, Tel: +90 50535576 00; Fax: +90 212 46070 70; E-mail: oguzkaraca@hotmail.com

Received June 30, 2015; Accepted July 30, 2015; Published August 01, 2015

Citation: Cakal B, Cakal SD, Karaca O, Omaygenc O, Boztosun O (2015) Juxtarenal Aortic Aneurysm Treated with Chimney Technique. J Vasc Med Surg 3: 212. doi:10.4172/2329-6925.1000212

Copyright: () 2015 Cakal B, et al. This is an open-access article distributed under the terms of the Creative Commons Attribution License, which permits unrestricted use, distribution, and reproduction in any medium, provided the original author and source are credited. 


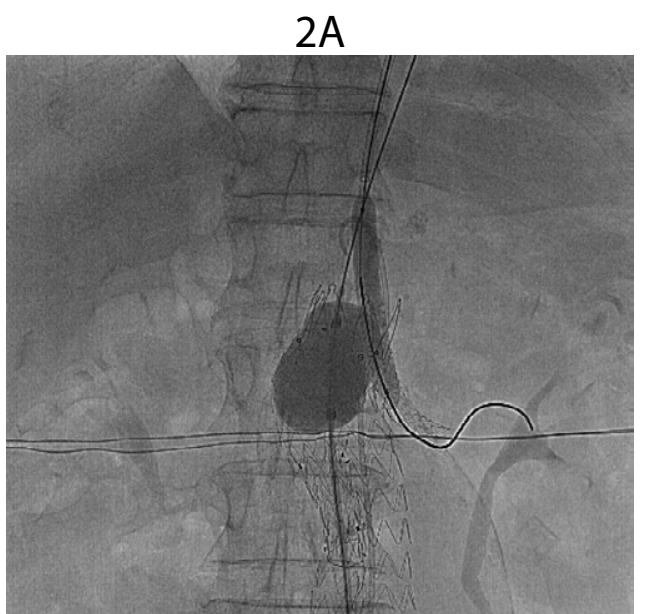

$2 \mathrm{~B}$

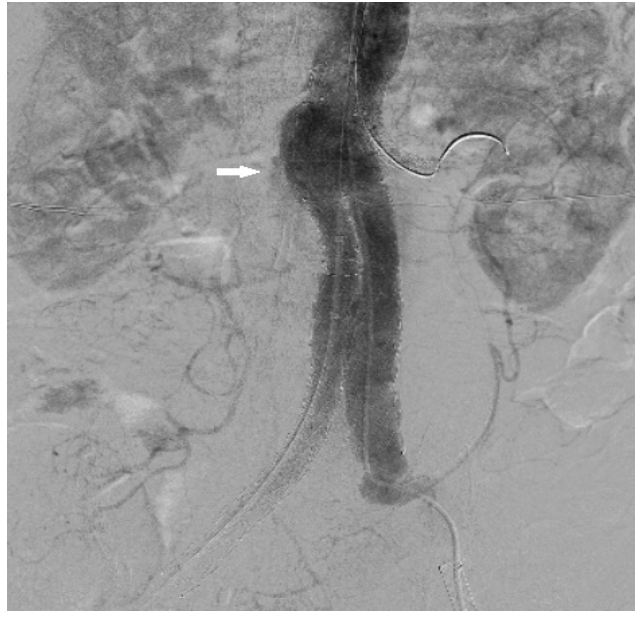

Figure 2: A: Synchronous balloon dilatation of the main body stent graft and renal stent graft. B: Postoperative angiography showed a slow-flow type $1 \mathrm{~A}$ endoleak (white arrow).

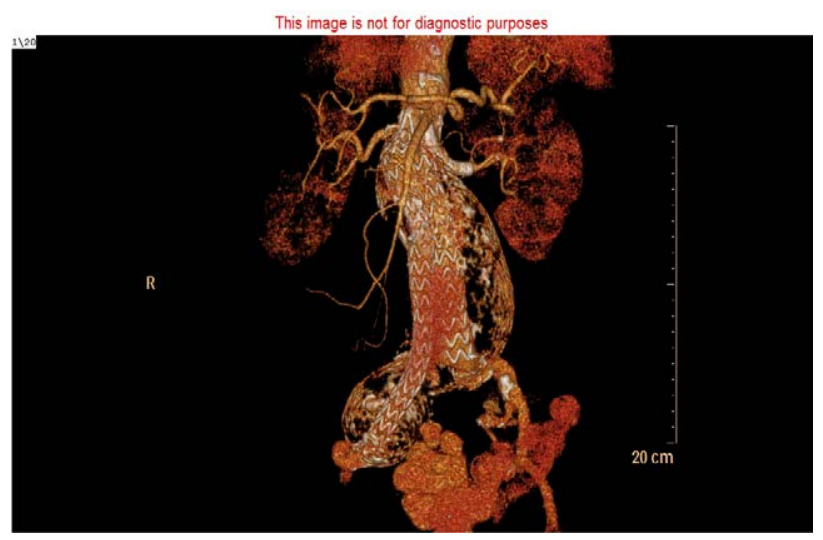

Figure 3: 3D and 2D CT angiography showing complete exclusion of the abdominal aortic aneurysm sac, spontaneous sealing of the endoleak and a patent stent graft deployed in the left renal artery at first month of the procedure.

when custom-made graft stent is not readily accessible. Complications during follow-up include occlusion of chimney stent or development of endoleak since a gap between the chimney stent and the aortic stent graft still exists. Short or mid-term results give promising results for chimney or parallel deployment techniques; lack of safety data for long term results limits its common use.

\section{References}

1. Lee JT, Greenberg JI, Dalman RL (2012) Early experience with the snorkel technique for juxtarenal aneurysms. J Vasc Surg 55: 935-946.

2. Ohrlander T, Snesson B, Ivancev K, Resch T, Dias N, et al. (2008) The chimney graft: a technique for preserving or rescuing aortic branch vessels in stent graft sealing zones. J Endovasc Ther 15: 427-432.

3. Hiramoto JS, Chang CK, Reilly LM, Schneider DB, Rapp JH, et al. (2009) Outcome of renal stenting for renal artery coverage during endovascular aortic aneurysm repair. J Vasc Surg 49: 1100-1106.

4. Scali ST, Feezor RJ, Chang CK, Waterman AL, Berceli SA, et al. (2014) Critical analysis of results after chimney endovascular aortic aneurysm repair raises cause for concern. J Vasc Surg 60: 865-873. 